\title{
Repetitive transcranial magnetic stimulation: an emerging treatment for medication-resistant depression
}

\author{
Jonathan Downar MD PhD, Daniel M. Blumberger MD MSc, Zafiris J. Daskalakis MD PhD
}

$\mathrm{M}$ ajor depressive disorder is a common, disabling and costly illness. At least one-third of patients with major depressive disorder do not respond to antidepressants or psychotherapy. ${ }^{1}$ Treatmentresistant depression (defined as failure of $\geq 2$ adequate medication trials) affects about $2 \%$ of Canadians, or 700000 individuals. ${ }^{1}$ New treatments are therefore urgently needed.

Novel brain-stimulation treatments are being used for an increasingly wide variety of neurologic and psychiatric disorders. Although several techniques are in development, one type in particular is currently transitioning from investigational to publicly funded clinical use in Canada: repetitive transcranial magnetic stimulation (rTMS).

\section{What is rTMS?}

Repetitive transcranial magnetic stimulation uses powerful, focused magnetic field pulses to induce electrical currents in target brain regions. The pulses are delivered via a hand-held or helmetshaped induction coil placed against the scalp over the target area (Figure 1). Single rTMS pulses are powerful enough to induce action potentials in the target region. Repeated trains of pulses cause changes in synaptic connections, via the mechanisms of neuroplasticity. Highfrequency stimulation $(5-20 \mathrm{~Hz})$ is considered excitatory and low-frequency stimulation $(1-5 \mathrm{~Hz})$ inhibitory. Multiple sessions of rTMS, delivered over several days, can produce durable increases or decreases in the activity of target brain regions, lasting weeks to months. ${ }^{2,3}$ Repetitive transcranial magnetic stimulation can thus normalize the activity of frontal lobe regions that are hyperactive or hypoactive in major depressive disorder. ${ }^{2}$

\section{How is it delivered?}

A therapeutic course of rTMS involves 20-30 sessions, usually delivered once daily on weekdays over four to six weeks, in an outpatient set- ting. Therapeutic rTMS is delivered by a trained technician or nurse, under physician supervision. Unlike with electroconvulsive therapy, no seizure is induced, and no anesthesia or activity restrictions are required. The conventional rTMS target in major depressive disorder is the dorsolateral prefrontal cortex, using high-frequency left-sided or low-frequency right-sided stimulation, or both. ${ }^{3,4}$ Other targets and protocols are also under investigation.

\section{Who is eligible?}

Patients with treatment-resistant depression (i.e., those who have not responded to antidepressant medications and/or psychotherapy) are potentially eligible for rTMS. Patients who have not tolerated antidepressant medications may also be eligible., ${ }^{3,5,6}$ Adult (18-65 yr) and geriatric $(>65 \mathrm{yr}$ ) populations are eligible; patients younger than 18 years are less well studied but are not considered ineligible. ${ }^{3,5}$ Patients should be screened for comorbid medical illnesses that can cause depressive symptoms (e.g., hypothyroidism and anemia). ${ }^{3,5}$ Ideally, patients should reside within commuting distance of the rTMS clinic because of the need for four to six weeks of treatment. For patients who are actively suicidal or too severely ill for outpatient treatment, electroconvulsive therapy offers higher remission rates. ${ }^{6}$
Competing interests: See end of article.

This article has been peer reviewed.

Correspondence to:

Jonathan Downar, jonathan. downar@uhn.ca

CMAJ 2016. DOI:10.1503 /cmaj.151316

\section{KEY POINTS}

- Repetitive transcranial magnetic stimulation (rTMS) is transitioning from investigational to clinical use as a novel therapeutic option for treatment-resistant depression.

- The treatment delivers trains of focused, magnetic field pulses to target brain regions over 20-30 daily sessions.

- Repetitive transcranial magnetic stimulation is safe and well-tolerated, and lacks the adverse cognitive effects of electroconvulsive therapy; adverse effects include transient scalp pain, headache and, rarely, seizure induction.

- Current protocols achieve $29 \%-49 \%$ response and $19 \%-34 \%$ remission in treatment-resistant depression, indicating intermediate efficacy between medication and electroconvulsive therapy. 
Repetitive transcranial magnetic stimulation is now available in 7 of 10 Canadian provinces, having gained Health Canada approval in 2002. Clinics accept referral in at least 13 major urban areas, comprising a total population of more than 17.5 million Canadians (for a directory, see Appendix 1, available at www.cmaj.ca/lookup/suppl/ doi:10.1503/cmaj.151316/-/DC1 or consult www. rtmscanada.ca). Treatment with rTMS is publicly covered in Quebec and Saskatchewan, and has been recommended for public coverage in Ontario and Alberta.

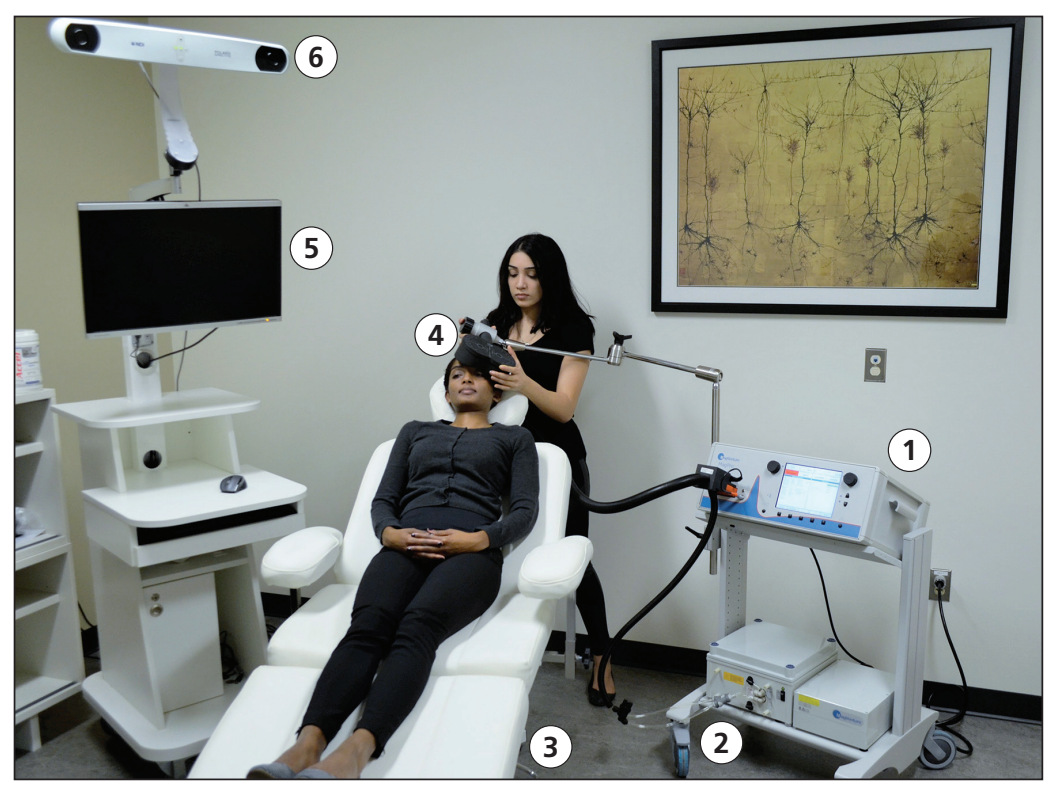

Figure 1: The elements of a repetitive transcranial magnetic stimulation (rTMS) treatment suite include the stimulator (1), cooling system (2), treatment chair (3), induction coil (4) and, in some cases, a magnetic resonance imaging (MRI)-guided navigation system (5) that incorporates a three-dimensional frameless stereotactic navigation camera (6). An rTMS technician or nurse typically performs the treatments. Cooling systems are not universally employed, but can allow for higher volumes of treatments per day; MRI-guided navigation systems are also not universally employed, but can offer greater accuracy for some applications of rTMS.

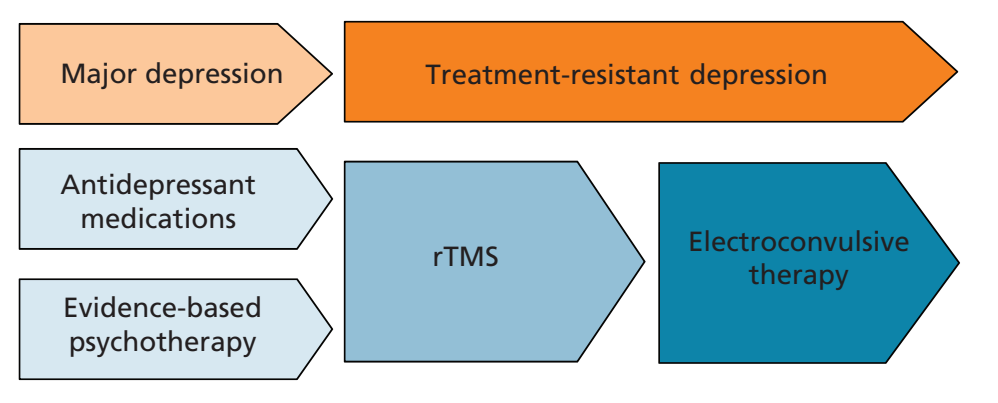

Figure 2: In the sequential treatment approach to major depression, repetitive transcranial magnetic stimulation (rTMS) begins to offer superior efficacy to additional medication trials or psychotherapy after the patient has had one to two adequate trials of medication without achieving remission. ${ }^{4,9}$ Electroconvulsive therapy offers superior efficacy to rTMS but is hampered by cognitive adverse effects, the need for anesthesia and substantially lower patient acceptance. ${ }^{6}$ Repetitive transcranial magnetic stimulation may therefore fill a therapeutically useful niche after medications and psychotherapy, but before electroconvulsive therapy, for patients with treatment-resistant depression.

\section{What are the possible harms?}

The most common adverse effects of rTMS are scalp pain during stimulation $(35 \%-40 \%)$, and transient headache after stimulation $(25 \%-30 \%))^{7}$ these symptoms diminish progressively over the treatment course and typically respond to over-thecounter analgesics. About $2 \%-4 \%$ of patients discontinue treatment because of pain. ${ }^{8}$ The all-causes discontinuation rate for rTMS is about $5 \%{ }^{8}$

Cases of rTMS-induced mania or hypomania have been reported, with an overall incidence of about $0.9 \%{ }^{3}$ Rare cases of rTMS-induced seizure have also been reported, with an overall incidence of less than $0.01 \% ;^{3}$ in one series of more than 10000 sessions, no seizures occurred. ${ }^{3}$ No cases of rTMS-kindled epilepsy have been reported.

Excitatory rTMS is generally considered contraindicated in patients with epilepsy and relatively contraindicated in patients with a history of seizure. Repetitive transcranial magnetic stimulation is also contraindicated in patients with intracranial foreign metal bodies or implanted devices (e.g., deep brain stimulator, cochlear implant, implanted medication pump, implanted cardiac defibrillator and pacemaker). ${ }^{3}$

Unlike with electroconvulsive therapy, rTMS effects on cognition appear benign. Meta-analyses report no significant evidence of impairment across a variety of neuropsychologic domains; some studies show significant cognitive improvement. ${ }^{7}$

\section{What is the evidence so far?}

The most widely cited recent meta-analysis of rTMS in major depression $(n=1371$ patients, 29 trials) reported $29.3 \%$ v. $10.4 \%$ response (odds ratio [OR] 3.3, 95\% confidence interval [CI] 2.354.64 ) and $18.6 \%$ v. $5.0 \%$ remission (OR $3.3,95 \%$ CI 2.04-5.32) for active v. sham rTMS. ${ }^{4}$ Another recent meta-analysis reported $29 \%$ v. $8 \%$ response (OR 3.38, 95\% CI 2.24-5.10; $n=643$ patients, 15 trials) and, in a separate sample, $30 \%$ v. $6 \%$ remission (OR 5.07, 95\% CI 2.50-10.30; $n=332$ patients, 7 trials) for active v. sham rTMS (mean difference in score on the Hamilton Depression Rating Scale 4.53, 95\% CI 2.96-6.11). ${ }^{9}$ The authors of the meta-analysis concluded that "for MDD [major depressive disorder] patients with 2 or more antidepressant treatment failures, rTMS is a reasonable, effective consideration." 9

In the United States, rTMS carries Food and Drug Administration (FDA) approval for major depressive disorder in patients who have not responded to one or more antidepressant trials. The 2007 study that supported FDA approval $(n=301)$ reported $24.5 \%$ v. $13.7 \%$ response (OR $2.23,95 \%$ CI $1.20-4.13$ ) and $15.5 \%$ v. $8.9 \%$ 
remission (OR 2.85, 95\% CI 1.23-6.63) for active v. sham rTMS (mean difference in score on the Hamilton Depression Rating Scale 2.8, $95 \%$ CI 1.2-4.4). ${ }^{10}$ A 2015 study that supported FDA approval for a helmet-shaped device for “deep" TMS $(n=212)$ reported $38.4 \%$ v. $21.4 \%$ response and $32.6 \%$ v. $14.6 \%$ remission for active v. sham rTMS (mean difference in score on the Hamilton Depression Rating Scale 3.11, 95\% CI 0.83-5.40). ${ }^{11}$

Regarding comparative clinical efficacy, a meta-analysis of head-to-head trials of electroconvulsive therapy v. rTMS ( $n=425$ patients, 9 trials) reported superior efficacy for electroconvulsive therapy at $64.4 \%$ v. $48.7 \%$ response (OR 1.41, 95\% CI $1.04-1.90$ ) and $52.9 \%$ v. $33.6 \%$ remission (OR $1.38,95 \%$ CI 1.10-1.74); ${ }^{6}$ both were superior to the remission rates of about $10 \%-20 \%$ reported for trials of additional medication or psychotherapy in treatment-resistant depression. ${ }^{12}$ Current evidence, therefore, positions rTMS efficacy slightly higher than that of psychotherapy and medications, but rather lower than electroconvulsive therapy, in a sequential treatment approach to major depressive disorder (Figure 2).

\section{What can we expect in the future?}

\section{Brief protocols}

Conventional rTMS sessions are lengthy (30-60 $\mathrm{min}$ ), limiting clinic capacity and increasing wait times. Newer protocols such as theta-burst stimulation require just one to three minutes. Studies currently underway will establish whether theta-burst stimulation protocols match or exceed conventional treatment efficacy, thus improving capacity.

\section{Accelerated courses}

Four to six weeks of once-daily stimulation is standard; however, some emerging research suggests that rTMS may be delivered multiple times per day, thereby substantially reducing the length of the course of treatment, while preserving response and remission rates. Future studies will establish whether rTMS courses can be completed in one to two weeks while preserving efficacy.

\section{References}

1. Nemeroff CB. Prevalence and management of treatment-resistant depression. J Clin Psychiatry 2007;68(Suppl 8):17-25.

2. Noda Y, Silverstein WK, Barr MS, et al. Neurobiological mechanisms of repetitive transcranial magnetic stimulation of the dorsolateral prefrontal cortex in depression: a systematic review. Psychol Med 2015;45:3411-32

3. Rossi S, Hallett M, Rossini PM, et al. Safety, ethical considerations, and application guidelines for the use of transcranial magnetic stimulation in clinical practice and research. Clin Neurophysiol 2009;120:2008-39.

4. Berlim MT, van den Eynde F, Tovar-Perdomo S, et al. Response, remission and drop-out rates following high-frequency repetitive transcranial magnetic stimulation (rTMS) for treating major depression: a systematic review and meta-analysis of randomized, doubleblind and sham-controlled trials. Psychol Med 2014;44:225-39.
5. Lefaucheur JP, André-Obadia N, Antal A, et al. Evidence-based guidelines on the therapeutic use of repetitive transcranial magnetic stimulation (rTMS). Clin Neurophysiol 2014;125:2150-206.

6. Ren J, Li H, Palaniyappan L, et al. Repetitive transcranial magnetic stimulation versus electroconvulsive therapy for major depression: a systematic review and meta-analysis. Prog Neuropsychopharmacol Biol Psychiatry 2014;51:181-9.

7. Loo CK, McFarquhar TF, Mitchell PB. A review of the safety of repetitive transcranial magnetic stimulation as a clinical treatment for depression. Int J Neuropsychopharmacol 2008;11:131-47.

8. Janicak PG, O'Reardon JP, Sampson SM, et al. Transcranial magnetic stimulation in the treatment of major depressive disorder: a comprehensive summary of safety experience from acute exposure, extended exposure, and during reintroduction treatment. J Clin Psychiatry 2008;69:222-32.

9. Gaynes BN, Lloyd SW, Lux L, et al. Repetitive transcranial magnetic stimulation for treatment-resistant depression: a systematic review and meta-analysis. J Clin Psychiatry 2014;75:477-89.

10. O'Reardon JP, Solvason HB, Janicak PG, et al. Efficacy and safety of transcranial magnetic stimulation in the acute treatment of major depression: a multisite randomized controlled trial. Biol Psychiatry 2007:62:1208-16.

11. Levkovitz Y, Isserles M, Padberg F, et al. Efficacy and safety of deep transcranial magnetic stimulation for major depression: a prospective multicenter randomized controlled trial. World Psychiatry 2015; 14:64-73.

12. Thase ME, Friedman ES, Biggs MM, et al. Cognitive therapy versus medication in augmentation and switch strategies as second-step treatments: a STAR*D report. Am J Psychiatry 2007;164:739-52.

Competing interests: Jonathan Downar has received research funding from the Canadian Institutes of Health Research (CIHR), Brain Canada, the National Institutes of Health (NIH), the Klarman Family Foundation, the Edgestone Foundation, and the Toronto General and Western Hospital Foundation, as well as travel stipends from Lundbeck and ANT Neuro, and in-kind equipment support for an investigator-initiated study from MagVenture. Daniel Blumberger receives research funding from CIHR, NIH, Brain Canada, the Temerty family through the Centre for Addiction and Mental Health (CAMH) Foundation and the Campbell Family Mental Health Research Institute. He receives nonsalary operating funds and in-kind equipment support from Brain Research and Development Services Ltd. for an investigator-initiated study. He is the site principal investigator for several sponsor-initiated clinical trials from Brain Research and Development Services Ltd. He received in-kind equipment support from Tonica and MagVenture for an investigator-initiated study. He received medication supplies for an investigator-initiated study from Invidior. In the last three years, Zafiris Daskalakis received research and equipment in-kind support for an investigator-initiated study through Brainsway and MagVenture. Zafiris Daskalakis has also served on the advisory board for Sunovion, Hoffmann-La Roche Limited and Merck and received speaker support from Eli Lilly. This work was supported by the Ontario Mental Health Foundation, CIHR, the Brain and Behaviour Research Foundation, and the Temerty family and Grant family through the CAMH Foundation and the Campbell Family Mental Health Research Institute.

Affiliations: Department of Psychiatry (Downar, Blumberger, Daskalakis); Institute of Medical Science (Downar, Blumberger, Daskalakis), University of Toronto, Toronto, Ont.; Krembil Research Institute (Downar); MRI-Guided Repetitive Transcranial Magnetic Stimulation Clinic (Downar), University Health Network, Toronto, Ont.; Temerty Centre for Therapeutic Brain Intervention and Campbell Family Mental Health Research Institute (Blumberger, Daskalakis), Centre for Addiction and Mental Health, Toronto, Ont.

Contributors: All of the authors contributed to the conception, literature review, writing and revision of the manuscript. Jonathan Downar prepared the figures and coordinated the writing, submission and revision of the manuscript. All of the authors gave final approval of the version to be published and agreed to act as guarantors of the work.

Acknowledgements: The authors gratefully acknowledge Dr. Peter Giacobbe, Dr. Fidel Vila-Rodriguez, Dr. Marcelo Berlim and Dr. Roumen Milev for their helpful comments and suggested revisions on previous versions of this manuscript. 